\title{
In vivo bioluminescence imaging of Burkholderia mallei respiratory infection and treatment in the mouse model
}

\author{
Shane Massey ${ }^{1}$, Katie Johnston ${ }^{1}$, Tiffany M. Mott' ${ }^{1}$, Barbara M. Judy ${ }^{2}$, Brian H. Kvitko ${ }^{3}$, \\ Herbert P. Schweizer ${ }^{3}$, D. Mark Estes ${ }^{4}$ and Alfredo G. Torres ${ }^{1,2,5 *}$ \\ 1 Department of Microbiology and Immunology, University of Texas Medical Branch, Galveston, TX, USA \\ 2 Department of Pathology, University of Texas Medical Branch, Galveston, TX, USA \\ ${ }^{3}$ Department of Microbiology, Immunology and Pathology, Colorado State University, Fort Collins, CO, USA \\ ${ }^{4}$ Department of Infectious Diseases, College of Veterinary Medicine, University of Georgia, Athens, GA, USA \\ ${ }^{5}$ Sealy Center for Vaccine Development and Center for Tropical Diseases, University of Texas Medical Branch, Galveston, TX, USA
}

\section{Edited by:}

Ivo Steinmetz, University of

Greifswald, Germany

\section{Reviewed by:}

Ifor Beacham, Griffith University, Australia

Paul J. Brett, University of South Alabama, USA

\section{*Correspondence:}

Alfredo G. Torres, Department of Microbiology and Immunology, University of Texas Medical Branch, 301 University Boulevard, Galveston, TX 77555-1070, USA.

e-mail: altorres@utmb.edu
Bioluminescent imaging (BLI) technology is a powerful tool for monitoring infectious disease progression and treatment approaches. BLI is particularly useful for tracking fastidious intracellular pathogens that might be difficult to recover from certain organs. Burkholderia mallei, the causative agent of glanders, is a facultative intracellular pathogen and has been classified by the CDC as a Category B select agent due to its highly infectious nature and potential use as a biological weapon. Very little is known regarding pathogenesis or treatment of glanders. We investigated the use of bioluminescent reporter constructs to monitor the dynamics of infection as well as the efficacy of therapeutics for $B$. mallei in real-time. A stable luminescent reporter $B$. mallei strain was created using the pUTmini-Tn 5::/uxKm2 plasmid and used to monitor glanders in the BALB/c murine model. Mice were infected via the intranasal route with $5 \times 10^{3}$ bacteria and monitored by BLI at 24,48 , and $72 \mathrm{~h}$. We verified that our reporter construct maintained similar virulence and growth kinetics compared to wild-type $B$. mallei and confirmed that it maintains luminescent stability in the presence or absence of antibiotic selection. The luminescent signal was initially seen in the lungs, and progressed to the liver and spleen over the course of infection. We demonstrated that antibiotic treatment $24 \mathrm{~h}$ post-infection resulted in reduction of bioluminescence that can be attributed to decreased bacterial burden in target organs. These findings suggest that $\mathrm{BLI}$ can be used to monitor disease progression and efficacy of therapeutics during glanders infections. Finally, we report an alternative method to mini-Tn $5:: / 4 \times \mathrm{Km} 2$ transposon using mini-Tn7-Iux elements that insert site-specifically at known genomic attachment sites and that can also be used to tag bacteria.

Keywords: bioluminescence, Burkholderia mallei, in vivo imaging, antibiotic

\section{INTRODUCTION}

Burkholderia mallei are Gram negative, facultative intracellular bacteria, and the causative agent for glanders. Horses, mules, and donkeys are the only known natural reservoirs. The disease has been eradicated in the United States, although it remains endemic in the Middle East, Central and South America, and Asia. Among humans, glanders is considered an occupational disease as most individuals who contract the disease are lab workers (Srinivasan et al., 2001) or individuals who come into close contact with infected animals (Sanford, 1995). Typically, human infections occur via contamination of the mucus membranes, wounds or abrasions. B. mallei is highly infectious in aerosol form, requiring very few organisms to establish an infection (Lever et al., 2003). B. mallei is also regarded as a potential biological threat due to their highly infectious nature and the incapacitating, often fatal disease progression in humans. Despite the history of $B$. malle $i$ as a bio-weapon (Sharrer, 1995; Christopher et al., 1997), little data about the efficacy of vaccinations or antibiotic treatment methods is available. Currently there are no vaccines available for either equine or human $B$. mallei infections. Numerous reports indicate in vitro susceptibility to a wide array of antibiotics (Kenny et al., 1999; Heine et al., 2001; Judy et al., 2009) including: aminoglycosides, cephalosporins, sulfonamides, macrolides, and fluoroquinolones; however, the in vivo efficacies are not well known. Due to the potential use as a biological weapon and the scarcity of in vivo treatment data, further evaluation of glanders progression in response to antibiotic challenge and/or vaccination is of utmost importance.

Bioluminescence imaging (BLI) is a technique based on the detection of visible light produced by luciferase-catalyzed reactions with a specific substrate. There are a variety of bioluminescent systems that have been identified in nature. The application of these as reporter systems can be divided into two strategies. The first one centers on the use of luciferase enzymes alone. Mammalian cells, or bacteria, can be engineered to express a plasmid-borne copy of the luciferase enzyme from organisms such as the firefly (Photinus pyralis) or the sea pansy (Renilla reniformis). Mammalian cells lack the required luciferin substrate for 
the bioluminescent reaction; therefore it must be exogenously supplied. The second strategy involves the use of the entire lux operon from a bacterium such as Photorhabdus luminescens (formerly Xenorhabdus luminescens). A transposon insertion or plasmid vector containing the luciferase enzyme, as well as the components for substrate production can be engineered with a transposon insertion (Flentie et al., 2008). This system will emit light in the range of 495-500 $\mathrm{nm}$. Since the enzyme and substrate are both synthesized, exogenous application of substrate is not necessary. The plasmid vectors or transposon insertions confer a constitutively expressed luminescent phenotype in a bacterial pathogen and permit the real-time in vivo monitoring of the organism. BLI has routinely been utilized to track disease progression in mice. Salmonella enterica serovar Typhimurium was transformed with the lux operon to determine virulence differences between three strains in one of the initial BLI studies on bacterial pathogenesis (Contag et al., 1995). BLI has since been used to monitor bacterial dissemination or to validate antimicrobial treatment regimen in Escherichia coli (Rocchetta et al., 2001; Foucault et al., 2010) and Mycobacterium tuberculosis (Andreu et al., 2010), as well as the select agents Francisella tularensis (Bina et al., 2010) and Burkholderia pseudomallei (Owen et al., 2009). Since bioluminescence is related to bacterial load, this property can be used to quantitatively assess the pathogen burden as well as to provide new insights into tissue tropism during infection (Hutchens and Luker, 2007).

Murine glanders has been widely used to study $B$. malle $i$ infections and potential therapeutics (Leakey et al., 1998; Fritz et al., 2000; Lever et al., 2003; Whitlock et al., 2008; Sarkar-Tyson et al., 2009). BALB/c mice are highly susceptible to B. mallei infection and provide an excellent model for acute glanders. C57BL/6 mice are more resistant to infection and are better suited for modeling chronic glanders. In this study, we monitored the bacterial infection in BALB/C mice with a strain of $B$. mallei containing a stable, chromosomally integrated copy of the lux operon from P. luminescens. After intranasal (i.n.) infection, the signal intensity associated with the bioluminescent bacteria increased over time in the lung as the bacteria replicated. Intraperitoneal antibiotic treatment correlated with decrease in bioluminescence, and the infection re-emerged after antibiotic treatment was stopped. This study demonstrates the potential for assessing therapeutic treatments using BLI.

\section{MATERIALS AND METHODS BACTERIAL STRAINS}

Escherichia coli S17-1 ( $\lambda$ pir) was used a host for the pUTminiTn5::luxKm2 transfer plasmid described by Winson et al. (1998). The plasmid was maintained in E. coli S17-1 ( $\lambda$ pir) and was mobilized via conjugation into B. mallei ATCC 23344 . For conjugation, E. coli S17-1 ( $\lambda$ pir) harboring the transfer plasmid and B. mallei ATCC 23344 were grown overnight at $37^{\circ} \mathrm{C}$ in $5 \mathrm{ml}$ Luria-Bertani (LB) media (the E. coli cultures was supplemented with $50 \mu \mathrm{g} / \mathrm{ml}$ kanamycin). One milliliter of each overnight culture was pelleted and washed with $1 \mathrm{ml} \mathrm{LB}$. Each culture was pelleted again and resuspended in $500 \mu \mathrm{l}$ of LB. The two cultures were combined and passed through a $0.45-\mu \mathrm{M}$ analytical filter. The filter was placed on an LB plate, which was incubated for $6 \mathrm{~h}$ at $37^{\circ} \mathrm{C}$. The cells were scraped from the surface of the filter and re-suspended in $3 \mathrm{ml}$ of phosphate buffered saline (PBS). Dilutions were spread onto LB plates supplemented with $4 \%$ glycerol (LBG) as well as $50 \mu \mathrm{g} / \mathrm{ml}$ kanamycin $(\mathrm{Km})$ and $50 \mu \mathrm{g} / \mathrm{ml}$ polymyxin B. These plates were then incubated at $37^{\circ} \mathrm{C}$ for $24 \mathrm{~h}$.

Bioluminescent colonies from the B. mallei lux strain, henceforth referred to as CSM001, were detected by an in vitro imaging system (IVIS) and were maintained on LBG plates with $\mathrm{Km}$. B. mallei strain ATCC 23344 was cultured on LBG for $48 \mathrm{~h}$ at $37^{\circ} \mathrm{C}$. The isolated colonies were cultured in LBG broth shaking for $24 \mathrm{~h}$ at $37^{\circ} \mathrm{C}$. Optical density readings $\left(\mathrm{OD}_{600}\right)$ were used to determine bacterial concentrations (CFU/ml). Bacteria were pelleted by centrifugation and re-suspended in LBG broth to obtain the desired concentration for inoculation.

Established protocols were used for isolation of bioluminescent B. thailandensis (Choi et al., 2005) and B. pseudomallei (Choi et al., 2005). Briefly, pUC18T-mini-Tn7T-Zeo-P1-lux was introduced in B. thailandensis strain E264R [a rifampicin resistant derivative of E264 (Choi et al., 2005)] by biparental mating using E. coli mobilizer strain SM10 ( $\lambda$ pir) and helper plasmid pTNS3 (Choi et al., 2008). Exconjugants were selected on Lennox LB medium (MO BIO Laboratories, Carlsbad, CA, USA) with $200 \mu \mathrm{g} / \mathrm{ml}$ zeocin (Invitrogen, Carlsbad, CA, USA) and $200 \mu \mathrm{g} / \mathrm{ml}$ rifampicin (Sigma, St. Louis, MO, USA). The pUC18T-miniTn7T-Gm-lux constructs, where the lux operon is expressed from endogenous promoters, were transferred to B. pseudomallei strain Bp340 (Mima and Schweizer, 2010) using biparental mating with E. coli mobilizer strain RHO3 (Lopez et al., 2009). Exconjugants were selected on LB Lennox medium with $15 \mu \mathrm{g} / \mathrm{ml}$ gentamicin (Sigma). The mini-Tn7 insertion sites were determined using colony PCR and previously described protocols (Choi et al., 2005, 2008). pUC18T-mini-Tn7T-Zeo-P1-lux is based on pUC18Tmini-Tn7T (Choi et al., 2006) and contains a zeocin resistance selection marker and the P. xenorhabdus luxCDABE operon from which internal restriction sites were removed (Voisey and Marincs, 1998). The lux operon on this plasmid is expressed from the P1 integron promoter (DeShazer and Woods, 1996).

All experiments conducted with B. mallei or B. pseudomallei were conducted using Select Agent compliant BSL-3/ABSL-3 containment and procedures.

\section{MICE}

Female, 6- to 8-week-old, BALB/c mice were obtained from Harlan (Indianapolis, IN, USA). Animal studies were performed in accordance with the Institutional Animal Care and Use Committee's guidelines at UTMB as recommended by the National Institute of Health.

\section{IN VITRO STABILITY}

The stability of the mini-Tn5::lux $\mathrm{Km} 2$ transposon insertion in $B$. malle $i$ was assessed by sub-culturing the CSM001 strain every $24 \mathrm{~h}$ over a period of 4 days as previously described (Foucault et al., 2010). Briefly, CSM001 was grown for $48 \mathrm{~h}$ on LBG agar plates. Isolated colonies were cultured in LBG broth shaking for $24 \mathrm{~h}$ at $37^{\circ} \mathrm{C}$. The overnight culture was diluted 1:1000 in LBG broth in the presence or absence of antibiotic and incubated with shaking for $24 \mathrm{~h}$ at $37^{\circ} \mathrm{C}$. Cells from the culture were plated on LBG plates in the presence and absence of antibiotic and incubated for $24-48 \mathrm{~h}$ at $37^{\circ} \mathrm{C}$. 
The plates were screened for bioluminescence and quantified using the IVIS Spectrum imaging system (Caliper Corp., Alameda, CA, USA). Each colony from the patch plate was selected as a region of interest (ROI). The Caliper Life Sciences software quantified the signal intensity for each ROI and an average intensity for each plate was calculated.

\section{GROWTH CURVE}

The growth curve for CSM001 $\left(\log _{10} \mathrm{CFU} / \mathrm{ml}\right)$ was performed in LBG broth and compared to the growth of wild-type B. mallei ATCC 23344 in three independent experiments. Sampling was performed at approximately $4 \mathrm{~h}$ intervals of the assay, from 0 to $16 \mathrm{~h}$, then at 26 and $38 \mathrm{~h}$ from the start of the experiment. The growth of B. mallei wild-type and CSM001 strains were monitored by plating serial dilutions from the time points on LBG media as well as by $\mathrm{OD}_{600}$ readings. Statistical differences were determined using an unpaired $t$ test with the significance set at $P<0.05$. The error bars represent the SEM.

\section{DETERMINATION OF LD $_{50}$}

Groups of 10 animals were i.n. inoculated with $10^{2}, 10^{3}, 10^{4}$, or $10^{5} \mathrm{CFUs}$ of CSM001 in a total volume of $50 \mu \mathrm{l} \mathrm{LB}$ broth ( $25 \mu \mathrm{l} /$ nare). Deaths were recorded over a period of 10 days. The $\mathrm{LD}_{50}$ was calculated (Reed and Muench, 1938) based on the dosages of the inocula.

\section{BACTERIAL CHALLENGE AND ANTIBIOTIC TREATMENT}

For the BLI experiments, animals were i.n. inoculated with $5 \times 10^{3}$ CFUs of CSM001, unless otherwise indicated, in a total volume of $50 \mu \mathrm{l}$ of LB broth $(25 \mu \mathrm{l} /$ nare $)$. Treatment with levofloxacin via the intraperitoneal (i.p.) route started $24 \mathrm{~h}$ after infection, once a day, up to $96 \mathrm{~h}$. Levofloxacin (Levaquin Injection, GlaxoSmithKline) was purchased from the UTMB Pharmacy and prepared according to the manufacturer's instructions at a dosage of $20 \mathrm{mg} / \mathrm{kg} /$ day.

\section{BACTERIAL COLONIZATION}

CSM001 was grown on LBG plates for $48 \mathrm{~h}$ at $37^{\circ} \mathrm{C}$ followed by sub-culturing in LBG broth shaking for $24 \mathrm{~h}$ at $37^{\circ} \mathrm{C}$. The bacterial culture was diluted to a concentration of $5 \times 10^{3} \mathrm{CFU}$ per $50 \mu \mathrm{l}$ (unless otherwise indicated). BALB/c mice were i.n. inoculated with the prepared dosage $(25 \mu \mathrm{l} /$ nare $)$. Animals were imaged and sacrificed at 24,48 , and $72 \mathrm{~h}$ post inoculation. The lungs, spleen, and liver (when indicated) were harvested. Bioluminescent signal was analyzed in the intact, extracted organs.

\section{BIOLUMINESCENCE QUANTIFICATION}

Bioluminescent images were acquired using an IVIS Spectrum (Caliper Corp., Alameda, CA, USA). This system consists of a heated stage inside a light-tight imaging chamber, a cooled chargecoupled-device camera with controller and refrigeration unit, integrated anesthesia system, and a Windows computer system for data acquisition and management. Bioluminescent signal was measured with no excitation (filters blocked) and an open emission filter.

In preparation for in vivo imaging, anesthesia was induced in the mice with $2-3 \%$ isoflurane in an oxygen-filled induction chamber. Once anesthetized, the mice were transferred to an isolation chamber, which was then placed in the imaging chamber and connected to the in-chamber anesthesia delivery system and maintained at $1-2 \%$ isoflurane. The ex vivo images of the lungs, spleen, and liver were acquired at each time point immediately after euthanasia. The organs were placed in a petri dish, and then contained in the isolation chamber (during imaging). Bioluminescent signal is represented in the images with a pseudocolor scale ranging from red (most intense) to violet (least intense) indicating the intensity of the signal. Scales were manually set to the same values for every comparable image (in vivo and ex vivo) to normalize the intensity of the bioluminescence across time points.

\section{RESULTS}

\section{ANALYSIS OF THE CSMO01 REPORTER STRAIN}

The CSM001 strain, a construct expressing the entire lux operon was used to avoid the exogenous addition of luciferase substrate. As a first step toward evaluating the use of bioluminescence for monitoring glanders, we wanted to ensure that the transposon integration was stable by utilizing an in vitro stability assay (Figure 1). CSM001 was grown in LBG and sub-cultured daily over a period of 4 days in the appropriate media. Bacteria were grown in the presence or absence of antibiotic to ensure that selection was not necessary to maintain the transposon stability. Every

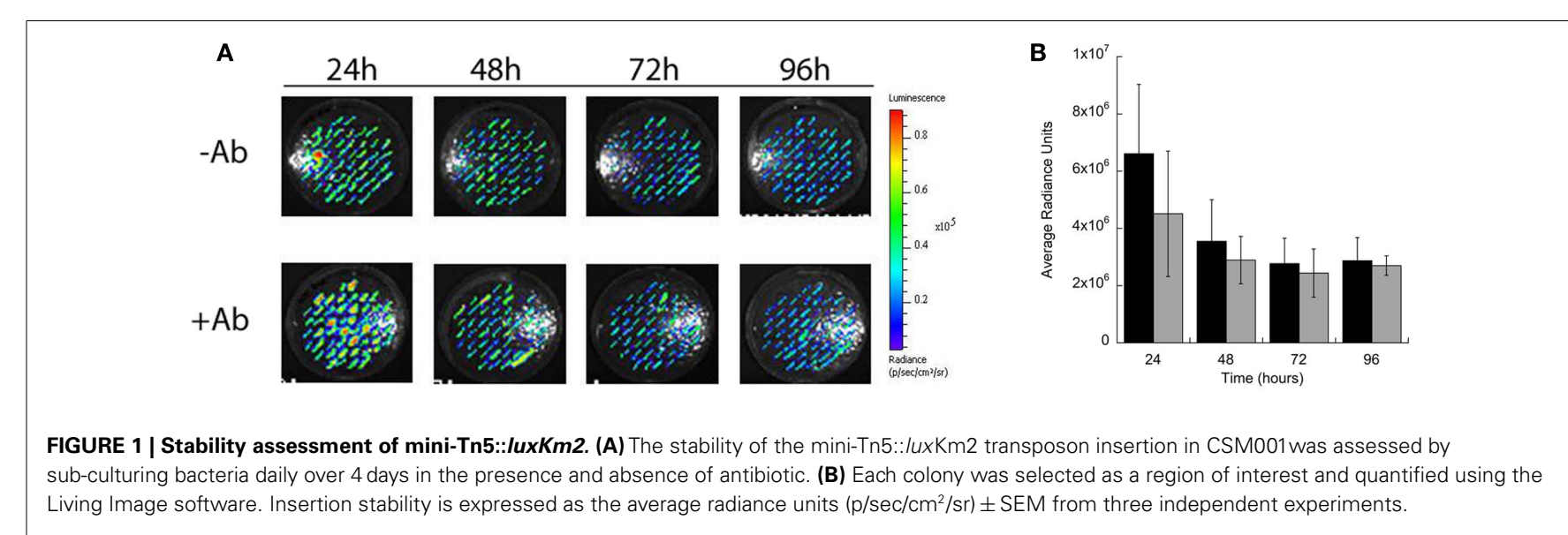


$24 \mathrm{~h}$, bacteria from the subculture were plated on the appropriate media, incubated at $37^{\circ} \mathrm{C}$ and monitored for bioluminescence. The bacteria did not exhibit any apparent difference in the bioluminescent signal on the patch plates (Figure 1A) following imaging. There was no significant decrease in the luminescent intensity over the 96-h time course (Figure 1B). The CSM001 strain sub-cultured in the presence of antibiotic exhibited the same level of luminescence as those sub-cultured in the absence of antibiotic.

Growth kinetic experiments were performed to ensure that $B$. mallei strain CSM001 grows comparably to the parental B. mallei ATCC 23344 strain. First we examined the virulence of our mutant strain compared to the wild-type strain (Figure 2A). Four groups of 10 animals were i.n. inoculated with $10^{2}-10^{5}$ CFUs of bacteria and monitored for survival over the 10-day time period. Following the Reed and Muench method (Reed and Muench, 1938), the 10 -day $\mathrm{LD}_{50}$ value was calculated to be $6.81 \times 10^{2} \mathrm{CFU}$. Next, we monitored the in vitro growth rate of the mutant strain compared to the wild-type strain (Figure 2B). Briefly, bacterial cultures were grown in LBG media over $38 \mathrm{~h}$. At the indicated time points the $\mathrm{OD}_{600}$ was determined and dilutions of the culture were grown on LBG plates. The growth curves indicate that the growth between the wild-type bacteria and the mutant strain is comparable and not significantly different throughout the time points within the exponential growth phase $(4-16 \mathrm{~h})$ stage.

\section{IN VIVO BIOLUMINESCENCE IMAGING}

Initial experiments were conducted to determine the threshold detection limits of the CSM001 strain. Groups of mice were i.n. infected with concentrations of bacteria ranging from $10^{2}$ to $10^{6}$ and monitored for daily luminescence analysis. Animals inoculated with the $10^{2}$ and $10^{3}$ doses of bacteria showed no apparent signs of infection 5 days post inoculation. Although luminescence was not detected in vivo following a 2-h incubation, a luminescent signal was detected in the lungs of animals infected with $1 \times 10^{6}$ bacteria as soon as $2 \mathrm{~h}$ post-infection, indicating a bacterial threshold for detection (Figure 3). A number of the animals infected at the higher doses exhibited luminescence signal associated within the brain, and all of these animals expired within $48 \mathrm{~h}$ after infection. No luminescence was initially detected with a dosage $5 \times 10^{3} \mathrm{CFU}$ (Figure 3) although the signal increased over time and the animals survived past $72 \mathrm{~h}$ (data not shown). We further tested whether the CSM001 strain could be used as a reporter strain to follow glanders progression in the BALB/C mouse model. Two groups of four BALB/c mice were infected i.n. with $5 \times 10^{3} \mathrm{CFUs}$ of the CSM001 strain (Figure 4). One group was anesthetized every $24 \mathrm{~h}$ and monitored for bioluminescence signal using the IVIS system, collecting, and quantifying the photons emitted by CSM001 within the animals. One animal was sacrificed every $24 \mathrm{~h}$, and the lungs and spleen were harvested for ex vivo imaging. Bioluminescence signal was absent at $24 \mathrm{~h}$ both in vivo and ex vivo, but was clearly evident at the 48 - and 72 -h time points. As predicted, the bioluminescent signal increased over the course of the study (Figure 4). CFU determinations confirmed that the observed bioluminescent signal increase. The bacterial burden within the lungs of untreated animals steadily increased from

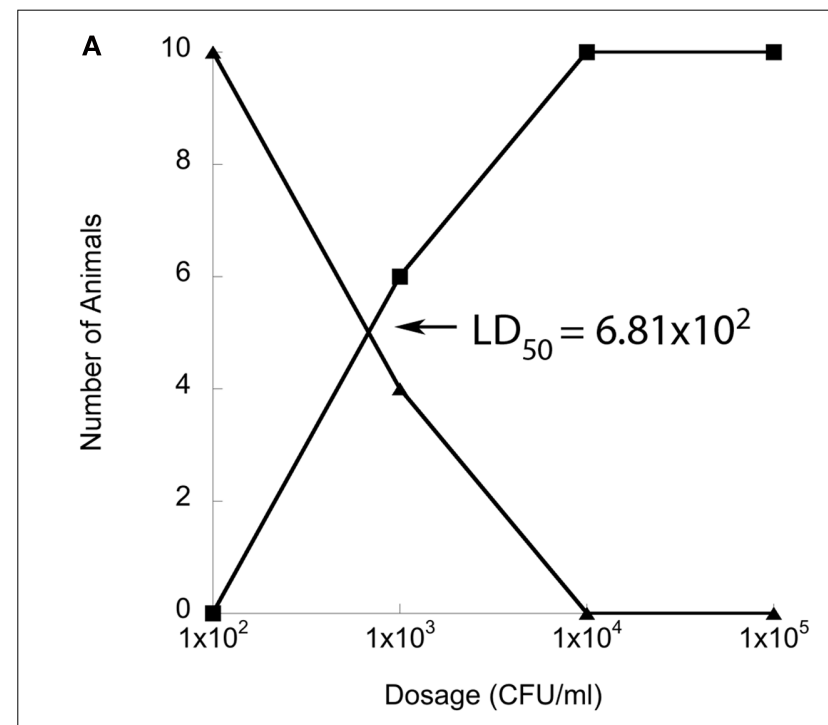

B

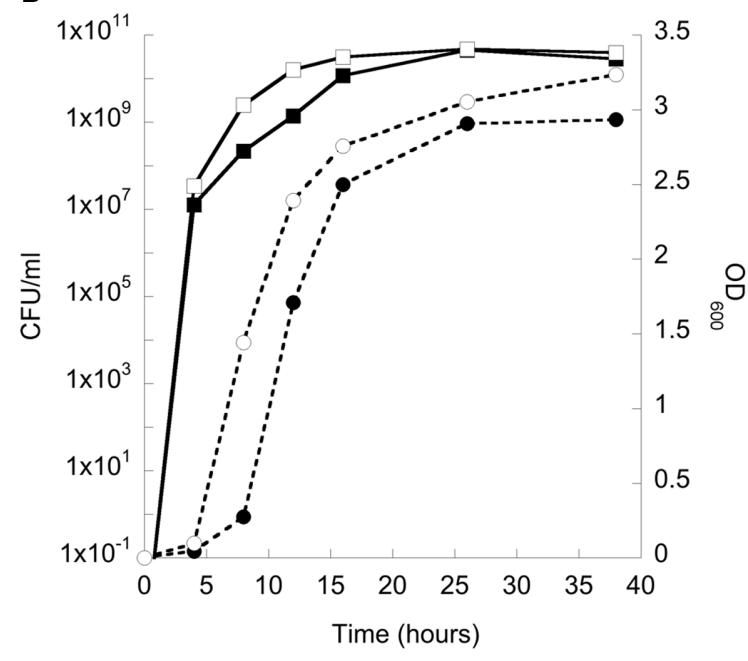

FIGURE 2 | CSM001 comparison to B. mallei ATCC 23344. (A) The 10-day $\mathrm{LD}_{50}$ for CSM001 was determined by infecting groups of 10 animals by i.n. with the indicated doses and monitoring the death (squares) and survival (triangles) of the animals. (B) Cultures of CSM001 (open symbols) and $B$. mallei ATCC 23344 (closed symbols) were grown to mid log phase ( $12 \mathrm{~h}$ ) while shaking at $37^{\circ} \mathrm{C}$. The cultures were diluted 1:100 and monitored by $\mathrm{OD}_{600}$ (circles) and CFU plating (squares) for $38 \mathrm{~h}$ at approximately $4 \mathrm{~h}$ intervals. Individual data points represent the CFU mean \pm SEM of three independent experiments. ${ }^{*}, P<0.05$.

approximately $7.77 \times 10^{5}$ at $24 \mathrm{~h}$ to $5.96 \times 10^{6}$ at $48 \mathrm{~h}$ and then to $3.17 \times 10^{8}$ at $72 \mathrm{~h}$ (Figure 6A). When the animals were treated with levofloxacin, the bacterial burden in the lungs was much lower $5.64 \times 10^{3}$ at $48 \mathrm{~h}$ and $1.59 \times 10^{5}$ at $72 \mathrm{~h}$ (Figure 6A). The bacterial burden within the spleen also increased over the course of the infection from approximately $2.15 \times 10^{4}$ at $24 \mathrm{~h}$ to $5.72 \times 10^{4}$ at $48 \mathrm{~h}$ and then $2.96 \times 10^{5}$ at $72 \mathrm{~h}$ (Figure 6B). Animals treated with levofloxacin also showed decreased bacterial burden in the spleen with $6.63 \times 10^{2} \mathrm{CFU} /$ organ at $48 \mathrm{~h}$ and $1.91 \times 10^{4} \mathrm{CFU} /$ organ at $72 \mathrm{~h}$ (Figure 6B). 


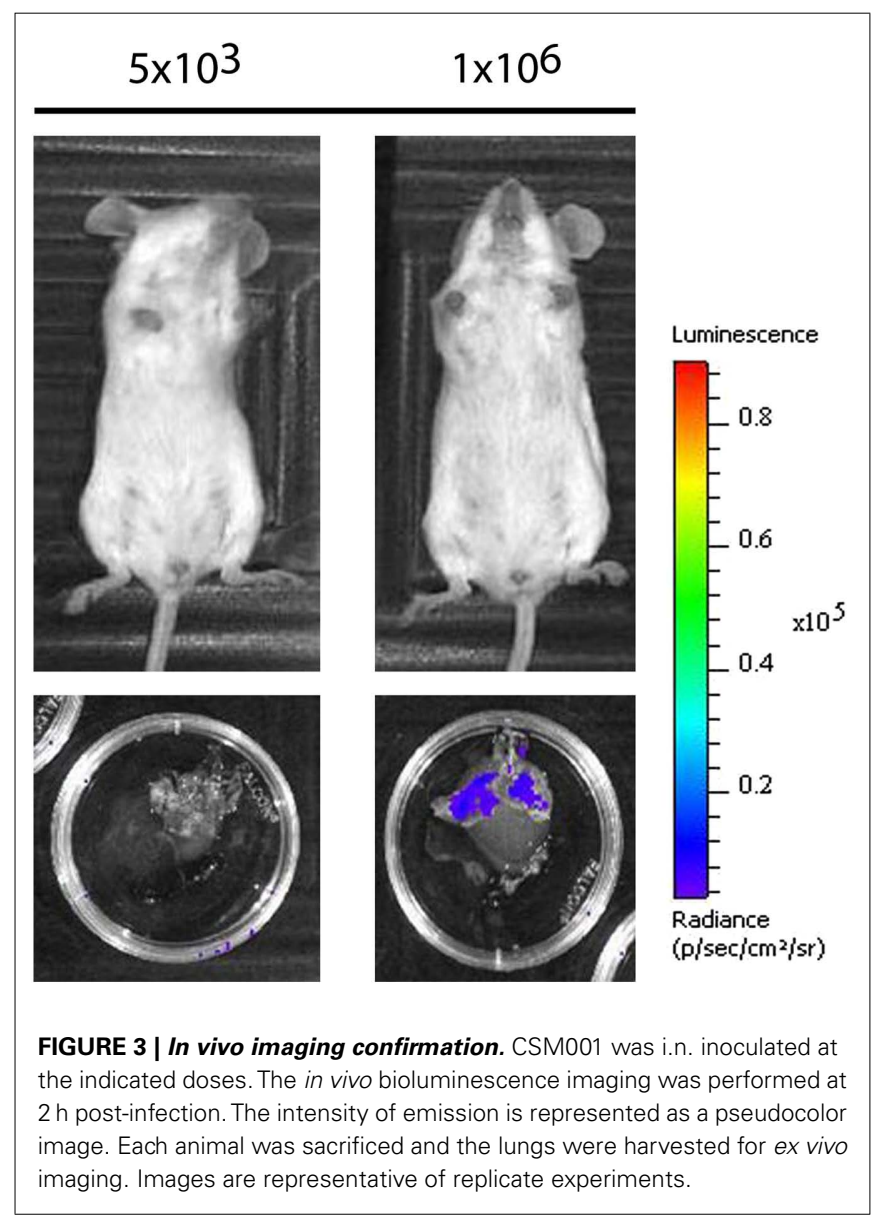

Our previous studies with the BALB/c mouse model have demonstrated that B. mallei is sensitive to levofloxacin both in vitro and in vivo (Judy et al., 2009). It was shown that treatment with levofloxacin decreased the bacterial burden in the lung and spleen of infected animals. Based on this evidence, we investigated whether the decrease in bacterial burden conferred by levofloxacin treatment could be detected with our bioluminescence system. A second group of animals (Figure 5A) received $20 \mathrm{mg} / \mathrm{kg} /$ day of levofloxacin $24 \mathrm{~h}$ after infection. Every $24 \mathrm{~h}$, the animals were anesthetized and monitored for bioluminescence signal. One animal was sacrificed for ex vivo imaging of the lung and spleen, as well as for CFU determination of those organs (data not shown). The levofloxacin treatment resulted in decreased bioluminescence signal over the course of the infection. At the 48-h time point, no signal can be detected in vivo or ex vivo although bacteria were recovered following organ plating (data not shown). A signal does appear following imaging at $72 \mathrm{~h}$ in both the ex vivo and in vivo images, however compared to the untreated animal the signal intensity is drastically reduced. This observation is consistent with our previous studies, as levofloxacin does not result in clearance of the bacterial infection. In the levofloxacin-treated animals, the antibiotic was discontinued after $96 \mathrm{~h}$. These animals were imaged every $24 \mathrm{~h}$ until a bioluminescence signal was detected. The re-emergence of signal occurred at $192 \mathrm{~h}$ and revealed apparent bacterial colonization in the brain (Figure 5B).

\section{CONSTRUCTION OF STABLE BIOLUMINESCENT BURKHOLDERIA STRAINS USING MINI-Tn7 TRANSPOSONS}

As an alternative to tagging with randomly inserting mini-Tn5-lux elements, we evaluated mini-Tn7-lux elements (Figure 7A) that insert site-specifically at known chromosomal attachment sites for tagging of Burkholderia spp. To this end, mini-Tn7-lux elements were integrated into the genomes of $B$. thailandensis strain E264 and B. pseudomallei strain Bp340. The resulting strains emitted light at levels that were comparable to those of $B$. mallei strain ATCC 23344 tagged with a randomly inserted mini-Tn5-lux element (Figure 1A). The levels of light emitted were somewhat dependent on the promoter used for lux operon expression. For example, the constitutive $\mathrm{P} 1$ integron promoter driving the $d h f R I I$ gene from R388 encoding trimethoprim resistance is six times more efficient in E. coli than the de-repressed tac promoter (DeShazer and Woods, 1996). Consequently, strong light emission was observed in B. thailandensis expressing the lux operon from this promoter (Figure 7B). By comparison, light emission by B. pseudomalle $i$ where the lux operon is preceded by an $\sim 500$ bp DNA fragment which contains at least five predicted (BDGP Neural Network Promoter Prediction; www.fruitfly.org/seq_tools/promoter.html) bacterial promoters with scores of 0.99 to 1.00 was still strong (Figure 7C) but less intense than that observed with $B$. thailandensis with a mini-Tn7-P1-lux element. Although several miniTn7-lux constructs with predicted strong promoters were tested in B. pseudomallei, cells containing the element with the five random promoters consistently exhibited stable, strong light expression. Promoter screening was by no means exhaustive and, for example, did not include the ompA promoter that was previously used to construct a mini-Tn7- $P_{o m p A}$-lux element that was successfully used to tag a $B$. pseudomallei strain and follow the disease manifestation in a murine melioidosis model (Owen et al., 2009).

\section{DISCUSSION}

Burkholderia mallei is a Category B select agent that has been used on a number of occasions as a biological weapon. Infection with $B$. mallei can result in glanders, a disease that affects horses and other equine species, developing into either an acute or chronic infection that ultimately leads to death. Glanders, while eradicated in many countries, remains endemic in a number of areas across the world. Despite the existence of endemic regions and potential threat of $B$. malle $i$ as a bio-weapon, little data is available on the efficacy of glanders treatments. Therefore, there is an urgent need for extensive evaluation of in vivo treatment methods. Data collected by other groups has demonstrated that in vivo bioluminescence imaging is a valuable tool for providing insights into the mechanisms of pathogenesis and disease progression, with the goal of identifying new therapeutic approaches. In this study, we demonstrate that glanders infection and treatment can be monitored using CSM001, a bioluminescent reporter strain derived from B. mallei ATCC 23344.

The CSM001 strain was created to monitor bacterial dissemination by constitutive expression of the lux operon from $P$. luminescens. The bacteria are constantly producing a luminescent signal that can be correlated to bacterial burden in affected organs. This simple and quantitative technique provides a number of advantages over current approaches for monitoring bacterial 


\section{$24 \mathrm{~h} \quad 48 \mathrm{~h} \quad 72 \mathrm{~h}$}
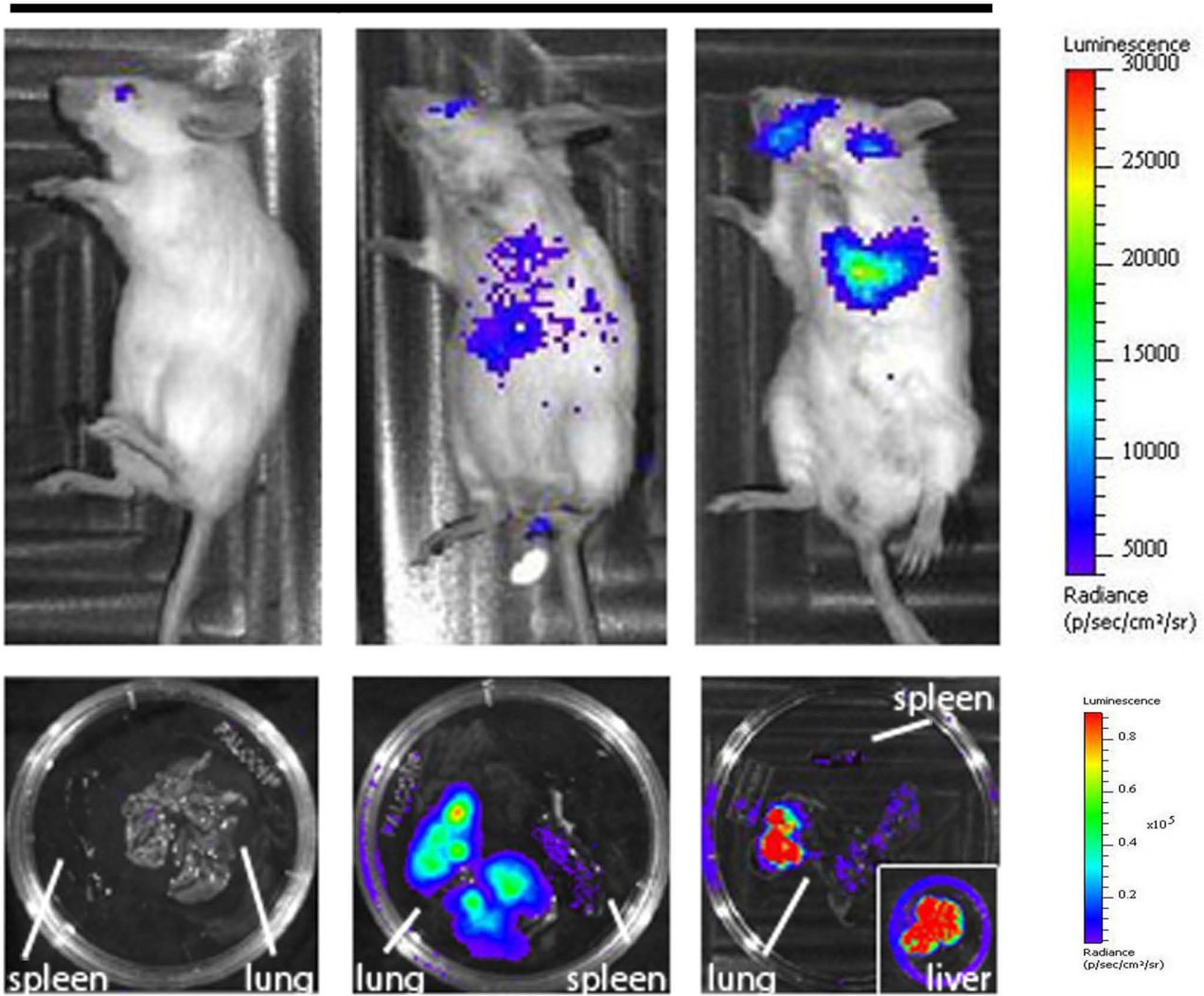

FIGURE 4 | In vivo imaging of CSM001 infection. CSM001 was i.n.

inoculated into mice. The in vivo bioluminescence imaging was conducted at 24,48 , and $72 \mathrm{~h}$ post-infection. The intensity of emission is represented as a pseudocolor image. At each time point, an animal was sacrificed and organs were harvested for ex vivo imaging and bacterial load determination. Images are representative of four replicate experiments. dissemination and therapeutic efficacies. Traditional methods for determining bacterial localization, colonization, and bacterial burden in vivo require that large numbers of experimental animals be euthanized at various time points for assessment of individual organs. The use of bioluminescence allows individual animals to be analyzed at multiple time points. This reduces animal-to-animal variation and ensures that biologically relevant information is not overlooked. In the conventional methods of harvesting organs and CFU plating, it is possible that the infection spreads to unexpected sites and that these infected tissues are not collected. BLI permits monitoring of the bacterial dissemination to these unexpected infection sites. The preferential use of bioluminescent reporters over fluorescent constructs is primarily due to the fact that most mammalian tissues are auto-fluorescent at the same wavelengths as the fluorescent reporter constructs, resulting in a low signal to noise ratio that is not observed when employing bioluminescent reporters.

Bioluminescent imaging has been used to study the mechanisms of pathogenesis and treatment efficacies for a number of infectious bacteria. One of the first investigations using BLI was conducted to monitor the distribution and differences in virulence among three strains of S. Typhimurium (Contag et al., 1995). In this study, the authors demonstrated the utility of the bioluminescence system by visualizing the efficacy of antibiotic treatment on infected animals. The in vivo bioluminescence has been utilized to detect $E$. coli localization in the neutropenic mouse thigh model where it was shown that bioluminescence was just as effective for determining antibiotic effectiveness as the traditional CFU determination methods (Rocchetta et al., 2001). Also, bioluminescence in $E$. coli has been used to track bacterial colonization in the mouse intestine (Foucault et al., 2010). BLI has also proven useful for monitoring bacterial dissemination in a number of other agents such as F. tularensis (Bina et al., 2010) and M. tuberculosis (Andreu et al., 2010). Even the colonization routes of B. pseudomallei, the closest relative of $B$. mallei, have been monitored using a lux-tagged strain (Owen et al., 2009).

Because the lux operon was inserted into the chromosome of $B$. malle $i$ via a transposon, it was important to ensure that insertion did not occur in a site that disrupted the virulence or the rate of growth of $B$. mallei. The $\mathrm{LD}_{50}$ assay and the growth curve analysis demonstrated that our CSM001 strain mirrored the virulence and growth kinetics of the B. mallei ATCC 23344 wild-type strain. If the transposon inserted into a site that increased virulence, it is possible that the infected mice would die prior to reaching a threshold 
A
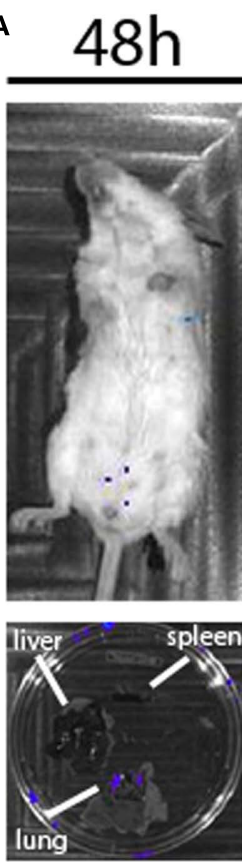
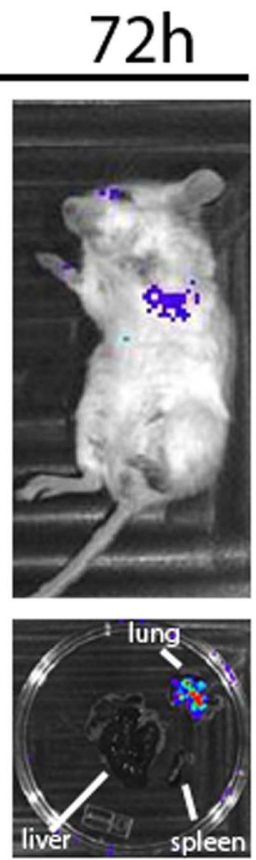

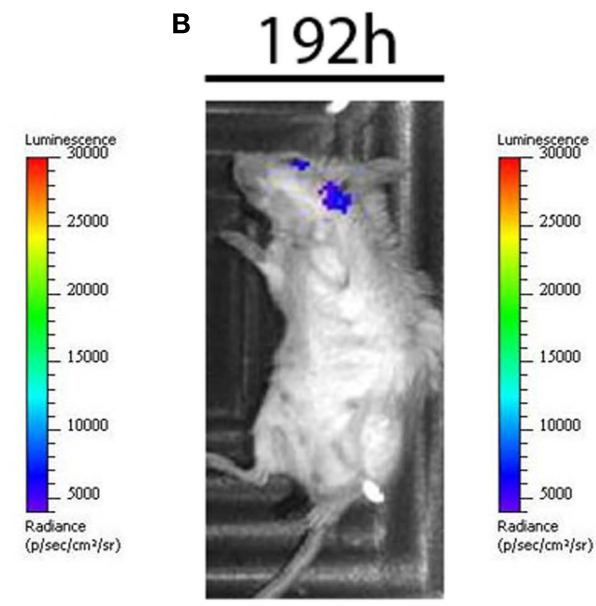

B
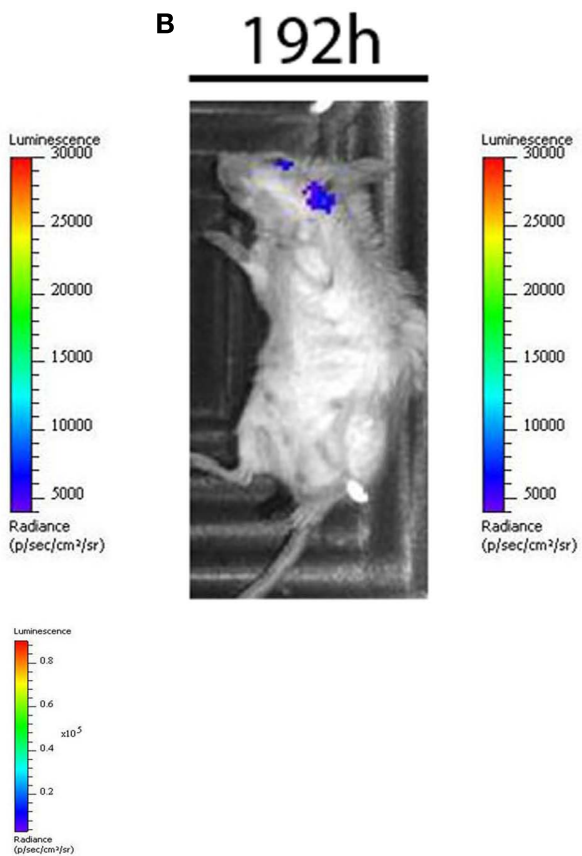

FIGURE 5 | In vivo imaging of CSM001 infection post-antibiotic treatment. (A) CSM001 was i.n. inoculated into mice. The in vivo bioluminescence was monitored at 24,48 , and $72 \mathrm{~h}$ post-infection. Treatment with $20 \mathrm{mg} / \mathrm{kg}$ of levofloxacin was administered after the 24-h time point. At each time point an animal was sacrificed and organs were harvested for ex vivo imaging and bacterial load determination. Images are representative of four replicate experiments. (B) Antibiotic treatment was suspended following $96 \mathrm{~h}$. Imaging was conducted every $24 \mathrm{~h}$ until the luminescence signal returned (192 $\mathrm{h}$ time point). detection limit. It has been previously reported that the $\mathrm{LD}_{50}$ for B. mallei ATCC 23344 ranges from $1.8 \times 10^{3}$ (Lever et al., 2003) to $2.5 \times 10^{5}$ (Whitlock et al., 2009). We determined the $\mathrm{LD}_{50}$ for the CSM001 strain to be $6.81 \times 10^{2}$. Our present study focused on the ability to create a bioluminescent $B$. mallei strain with similar virulence and growth kinetics to the wild-type strain.

As an alternative to tagging with randomly inserting miniTn5-lux transposons, mini-Tn7-lux elements that insert sitespecifically at known chromosomal attachment sites can be used to construct reporter bacteria. The attachment sites are generally located in intergenic regions and thus integrants are less likely to be attenuated. Mini-Tn7 elements offer the added advantage that, unlike "locked in" mini-Tn5 insertions, they can be readily inserted at the same site in mutant derivatives of the same bacterial strain thereby allowing comparative analyses in isogenic backgrounds. While mini-Tn7-lux derivatives have been used to construct bioluminescent B. pseudomallei and B. thailandensis strains (Figure 7; Owen et al., 2009), they have not yet been employed for construction of bioluminescent B. mallei. However, this will be easy to achieve since detailed protocols for site- and orientation-specific mini-Tn7 tagging of Burkholderia spp. have been published, including B. thailandensis (Choi et al., 2005), B. pseudomallei (Choi et al., 2008), and B. mallei (Choi et al., 2006).

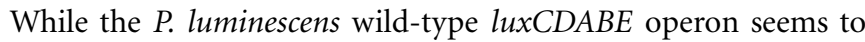
be well expressed in Burkholderia spp., there is room for improvement. Stable expression of the lux operon can be further optimized by screening of additional promoters known to be well-tolerated and constitutively expressed in Burkholderia spp., for example the s12 ribosomal gene promoter (Choi et al., 2008) or the ompA gene promoter (Owen et al., 2009). The P. luminescens wild-type lux$C D A B E$ operon is also very $\mathrm{A}+\mathrm{T}$ rich $(>69 \%)$ and it has been proposed that for this and other reasons, e.g., rare codon usage, these genes may not be expressed very well in bacteria with high $\mathrm{G}+\mathrm{C}$ content genomes. To overcome this potential hurdle, Craney et al. (2007) derived a synthetic luxCDABE gene cluster optimized for expression in bacteria with high $\mathrm{G}+\mathrm{C}$ content. It may be worthwhile considering engineering of mini-Tn7-lux elements that exhibit strong, yet stable, light emission using a combination of optimized genus- or species-specific promoter sequences and a lux operon optimized for gene expression in bacteria with high $\mathrm{G}+\mathrm{C}$ content.

The in vivo BLI can be used to identify dissemination of bacterial infections in the i.n. mouse model. The BALB/c mouse model has routinely been used to study B. mallei infection, as it is highly susceptible (Leakey et al., 1998; Fritz et al., 2000; Lever et al., 2003; Whitlock et al., 2008; Sarkar-Tyson et al., 2009). We monitored BALB/c mice that had been i.n. infected with the CSM001 strain. Over the course of the infection, the bacterial burden in the target organs increased and the infection sites became luminescent and visible with the IVIS. The data collected are consistent with the traditional methods for characterizing bacterial infection. Lever et al. (2003) demonstrated that following aerosol exposure to $B$. mallei, bacterial counts are first detected in the lungs and steadily increased over 5 days, at which point the bacterial 


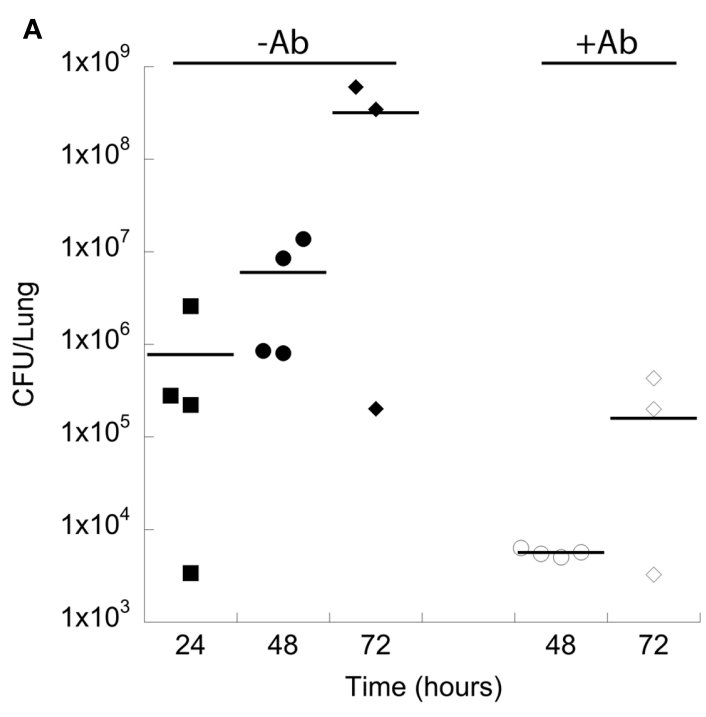

B

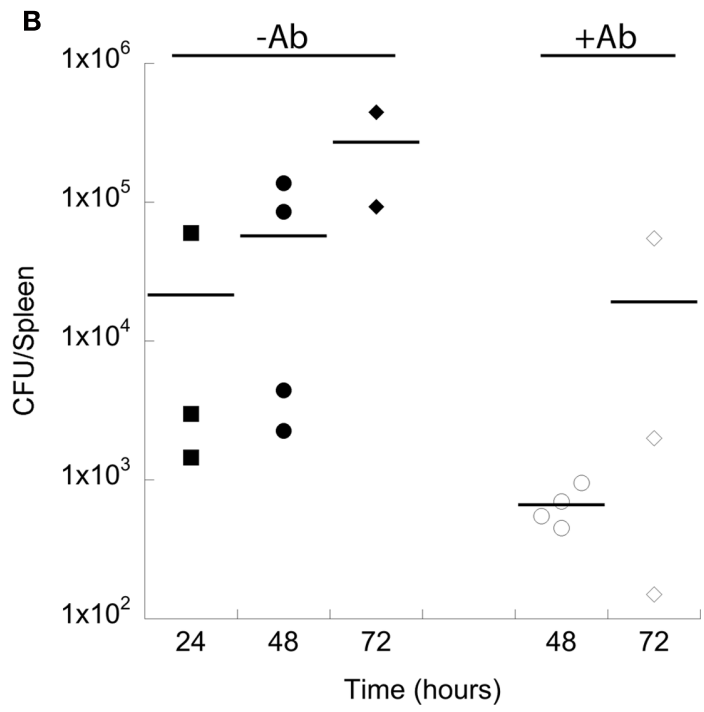

FIGURE 6 | B. mallei bacterial burden in treated and untreated $\boldsymbol{B A B L B / c}$ mice. CSM001 was i.n. inoculated into BALB/C mice as indicated previously for the bioluminescent imaging. At each time point, untreated (closed symbols) or treated (open symbols) animals with $20 \mathrm{mg} / \mathrm{kg} /$ day of levofloxacin were sacrificed and the lungs (A) and spleen (B) were harvested at $24 \mathrm{~h}$ (squares), $48 \mathrm{~h}$ (circles), $72 \mathrm{~h}$ (diamonds) and plated for CFU determination.

burden decreases. We showed that after $24 \mathrm{~h}$ of infection there is no bioluminescence signal in the lungs, despite the presence of approximately $1 \times 10^{6}$ bacteria. Presumably this is because a threshold detection limit has not been reached as is evident in Figure 3. After $48 \mathrm{~h}$, the bioluminescence signal is visible and this signal increases in intensity at $72 \mathrm{~h}$ (Figure 4).

Currently, there are no effective vaccines available for glanders infection and very little data exists on the efficacy of antibiotic for treatment of human glanders. One case of human glanders involving an accidental lab exposure was treated with a mixed regimen of antibiotics (Srinivasan et al., 2001). A more standardized course of treatment is necessary however, due to the fact that

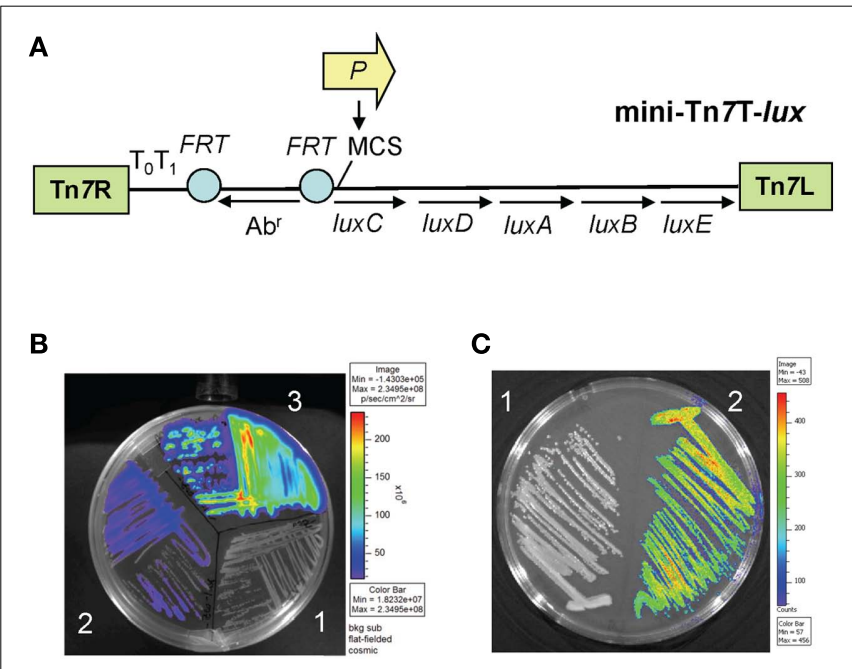

FIGURE 7 | Bioluminescent tagging of Burkholderia spp. with mini-Tn 7-Iux elements. (A) Map of a typical mini-Tn 7-lux element. The basic element contains a promoter-less $P$. luminescens luxCDABE operon that is preceded by a multiple cloning site (MCS). An antibiotic resistance ( $A b^{r}$ ) selection marker inserted upstream of the lux operon is preferably flanked by yeast Flp recombinase target (FRT) sites that allow optional site-specific excision of the $A b^{r}$ marker after site-specific insertion into the bacterial chromosome. Restriction sites in the MCS can also be used to insert customized promoter sequences (arrow labeled P). (B) A mini-Tn 7-lux element with a zeocin resistance selection marker was inserted into the $\mathrm{g} / \mathrm{mS} 1$-associated insertion site of chromosome 1 of $B$. thailandensis strain E264 (Choi et al., 2005). Bacteria containing either no insertion (1), a promoter-less mini-Tn7-lux (2) or a mini-Tn7-P1-lux element where the lux operon is transcribed from the strong P1 integron promoter (DeShazer and Woods, 1996) were grown overnight on an LB plate and imaged on an IVIS Spectrum imaging system. (C) A mini-Tn 7-lux element with a gentamicin resistance selection marker was inserted into the g/mS3-associated insertion site on chromosome 2 of B. pseudomallei strain Bp340 (Choi et al. 2008; Mima and Schweizer, 2010). Bacteria containing either no insertion (1) or a mini-Tn7-lux element where the lux operon is transcribed from synthetic promoter sequences active in B. pseudomallei (2) were grown overnight on an LB plate and imaged on an IVIS Spectrum imaging system.

mixed antibiotic regimen must be administered for a number of days and assumes an accurate and early diagnosis. The antibiotic susceptibility of $B$. malle $i$ is similar to that of $B$. pseudomalle $i$ and because of this, most $B$. mallei antibiotic treatments are based on efficacy of treatment regimens for B. pseudomallei infections. Levofloxacin inhibits DNA replication and transcription in Gram negative bacteria and has previously been shown to decrease bacterial burden in the lung and spleen of $\mathrm{BALB} / \mathrm{c}$ mice infected with $B$. mallei (Judy et al., 2009). Here, we demonstrated that the decrease in bacterial burden in the lungs can be visualized using BLI technology. Animals were treated with antibiotic $24 \mathrm{~h}$ post-infection, and when the animals were imaged at $72 \mathrm{~h}$, a luminescence signal was visible, although the intensity was markedly reduced. Antibiotic efficacy studies have revealed that $B$. mallei is susceptible to a number of antibiotics in vitro, although in vivo analysis still needs to be performed; therefore, BLI is a useful tool for assessing various treatments using fewer animals. BLI should also prove useful for monitoring novel therapeutic targets such as new antibiotics, quorum sensing molecules (reviewed in Estes et al., 2010) or virulence 
factors associated with the type three (Whitlock et al., 2007) and type six secretion systems (Schell et al., 2007).

A number of our BLI experiments revealed bioluminescence signal associated with the brain of the animals. It was previously reported that the nasal-associated lymphoid tissue (NALT) of $\mathrm{BALB} / \mathrm{c}$ mice acts as an entryway for B. pseudomallei (Owen et al., 2009). The authors concluded that the olfactory nerve served as a route of entry for B. pseudomallei to the brain after i.n. infection with $3.6 \times 10^{5} \mathrm{CFU}$. The implication was also made that this route of entry could be responsible for the infections of the CNS often seen in human melioidosis (Currie et al., 2000; Chadwick et al., 2002; White, 2003; Koszyca et al., 2004). Based on our observations (Figure 5B), it is possible that B. mallei utilizes the same olfactory nerve to reach the brain in mice. Owen et al. (2009) observed localization of B. pseudomallei to the brain $48 \mathrm{~h}$ after infection. Our experiments with $B$. mallei required at least $72 \mathrm{~h}$ before signal could be detected. Further clarification of the role of NALT during glanders infection could provide insights for future vaccination therapies.

In summary, we have developed and validated a BLI technique for the detection of $B$. mallei infection in the BALB/C intranasal model of acute glanders. We demonstrated that this

\section{REFERENCES}

Andreu, N., Zelmer, A., Fletcher, T., Elkington, P. T., Ward, T. H., Ripoll, J., Parish, T., Bancroft, G. J., Schaible, U., Robertson, B. D., and Wiles, S. (2010). Optimisation of bioluminescent reporters for use with mycobacteria. PLoS ONE 5, e10777. doi: 10.1371/journal.pone.0010777

Bina, X. R., Miller, M. A., and Bina, J. E. (2010). Construction of a bioluminescence reporter plasmid for Francisella tularensis. Plasmid 64, 156-161.

Chadwick, D. R., Ang, B., Sitoh, Y. Y., and Lee, C. C. (2002). Cerebral melioidosis in Singapore: a review of five cases. Trans. R. Soc. Trop. Med. Hyg. 96, 72-76.

Choi, K. H., Deshazer, D., and Schweizer, H. P. (2006). mini-Tn7 insertion in bacteria with multiple glmS-linked att $\operatorname{Tn} 7$ sites: example Burkholderia mallei ATCC 23344. Nat. Protoc. 1, 162-169.

Choi, K. H., Gaynor, J. B., White, K. G., Lopez, C., Bosio, C. M., KarkhoffSchweizer, R. R., and Schweizer, H. P. (2005). A Tn7-based broad-range bacterial cloning and expression system. Nat. Methods 2, 443-448.

Choi, K. H., Mima, T., Casart, Y., Rholl, D., Kumar, A., Beacham, I. R., and Schweizer, H. P. (2008). Genetic tools for select-agent-compliant manipulation of Burkholderia pseudomallei. Appl. Environ. Microbiol. 74, 1064-1075.

Christopher, G. W., Cieslak, T. J., Pavlin, J. A., and Eitzen, E. M. Jr. (1997).
Biological warfare. A historical perspective. JAMA 278, 412-417.

Contag, C. H., Contag, P. R., Mullins, J. I., Spilman, S. D., Stevenson, D. K., and Benaron, D. A. (1995). Photonic detection of bacterial pathogens in living hosts. Mol. Microbiol. 18, 593-603.

Craney, A., Hohenauer, T., Xu, Y., Navani, N. K., Li, Y., and Nodwell, J. (2007). A synthetic luxCDABE gene cluster optimized for expression in high-GC bacteria. Nucleic Acids Res. 35, e46.

Currie, B. J., Fisher, D. A., Howard, D. M., and Burrow, J. N. (2000). Neurological melioidosis. Acta Trop. 74, 145-151.

DeShazer, D., and Woods, D. E. (1996). Broad-host-range cloning and cassette vectors based on the R388 trimethoprim resistance gene. Biotechniques 20, 762-764.

Estes, D. M., Dow, S. W., Schweizer, H. P., and Torres, A. G. (2010). Present and future therapeutic strategies for melioidosis and glanders. Expert Rev. Anti. Infect. Ther. 8, 325-338.

Flentie, K. N., Qi, M., Gammon, S. T., Razia, Y., Lui, F., Marpegan, L., Manglik, A., Piwnica-Worms, D., and Mckinney, J. S. (2008). Stably integrated luxCDABE for assessment of Salmonella invasion kinetics. Mol. Imaging 7, 222-233.

Foucault, M. L., Thomas, L., Goussard, S., Branchini, B. R., and Grillot-Courvalin, C. (2010). In vivo bioluminescence imaging for the

highly sensitive reporter strategy can be used to obtain in vivo images of B. mallei infection in mice. This BLI method is straightforward and reduces the numbers of animals traditionally required and can be useful for antibiotic efficacy studies as shown by the decrease in luminescent signal of levofloxacin-treated animals. Future studies will verify previously reported in vitro antibiotic efficacies and test vaccine candidates.

\section{ACKNOWLEDGMENTS}

We thank Dr. Richard Titball from the University of Exeter for the generous gift of the bioluminescent transposon and to Dr. Sonja Lloyd and Dr. Douglas Botkin for critical reading of the manuscript. Grant number U54 AI057156 from the Western Regional Center for Excellence supported this project along with grant number 5U01AI082103-02 and a T32 postdoctoral training grant, number T32A1007536, from the NIAID/NIH. Herbert P. Schweizer was supported by grant AI065357 from the NIAID/NIH. Herbert P. Schweizer acknowledges the contributions of K.-H. Choi, A. Kumar, and D. A. Rholl to work performed with mini-Tn7-lux in his laboratory. The contents are solely the responsibility of the authors and do not necessarily represent the official views of the RCE Programs Office, NIAID, or NIH.

study of intestinal colonization by Escherichia coli in mice. Appl. Environ. Microbiol. 76, 264-274.

Fritz, D. L., Vogel, P., Brown, D. R., Deshazer, D., and Waag, D. M. (2000). Mouse model of sublethal and lethal intraperitoneal glanders (Burkholderia mallei). Vet. Pathol. 37, 626-636.

Heine, H. S., England, M. J., Waag, D. M., and Byrne, W. R. (2001) In vitro antibiotic susceptibilities of Burkholderia mallei (causative agent of glanders) determined by broth microdilution and E-test. Antimicrob. Agents Chemother. 45, 2119-2121.

Hutchens, M., and Luker, G. D. (2007). Applications of bioluminescence imaging to the study of infectious diseases. Cell. Microbiol. 9, 2315-2322.

Judy, B. M., Whitlock, G. C., Torres, A. G., and Estes, D. M. (2009). Comparison of the in vitro and in vivo susceptibilities of Burkholderia mallei to ceftazidime and levofloxacin. BMC Microbiol. 9, 88. doi: 10.1186/14712180-9-88

Kenny, D. J., Russell, P., Rogers, D., Eley, S. M., and Titball, R. W. (1999). In vitro susceptibilities of Burkholderia mallei in comparison to those of other pathogenic Burkholderia spp. Antimicrob. Agents Chemother. 43, 2773-2775.

Koszyca, B., Currie, B. J., and Blumbergs, P. C. (2004). The neuropathology of melioidosis: two cases and a review of the literature. Clin. Neuropathol. 23, 195-203.
Leakey, A. K., Ulett, G. C., and Hirst, R. G. (1998). BALB/C and C57Bl/6 mice infected with virulent Burkholderia pseudomallei provide contrasting animal models for the acute and chronic forms of human melioidosis. Microb. Pathog. 24, 269-275.

Lever, M. S., Nelson, M., Ireland, P. I., Stagg, A. J., Beedham, R. J., Hall, G. A., Knight, G., and Titball, R. W. (2003). Experimental aerogenic Burkholderia mallei (glanders) infection in the BALB/C mouse. J. Med. Microbiol. 52, 1109-1115.

Lopez, C. M., Rholl, D. A., Trunck, L. A., and Schweizer, H. P. (2009). Versatile dual-technology system for markerless allele replacement in Burkholderia pseudomallei. Appl. Environ. Microbiol. 75, 6496-6503.

Mima, T., and Schweizer, H. P. (2010). The BpeAB-OprB efflux pump of Burkholderia pseudomallei 1026b does not play a role in quorum sensing, virulence factor production, or extrusion of aminoglycosides but is a broad-spectrum drug efflux system. Antimicrob. Agents Chemother. 54, 3113-3120.

Owen, S. J., Batzloff, M., Chehrehasa, F., Meedeniya, A., Casart, Y., Logue, C. A., Hirst, R. G., Peak, I. R., Mackay-Sim, A., and Beacham, I. R. (2009). Nasal-associated lymphoid tissue and olfactory epithelium as portals of entry for Burkholderia pseudomallei in murine melioidosis. J. Infect. Dis. 199, 1761-1770. 
Reed, L. J., and Muench, H. (1938). A simple method of estimating fifty per cent endpoints. Am. J. Hygiene 27, 493-497.

Rocchetta, H. L., Boylan, C. J., Foley, J. W., Iversen, P. W., Letourneau, D. L., Mcmillian, C. L., Contag, P. R., Jenkins, D. E., and Parr, T. R. Jr. (2001). Validation of a noninvasive, real-time imaging technology using bioluminescent Escherichia coli in the neutropenic mouse thigh model of infection. Antimicrob. Agents Chemother. 45, 129-137.

Sanford, J. P. (1995). "Pseudomonas species (including melioidosis and glanders)," in Principles and Practice of Infectious Diseases, 4th Edn, eds G. L. Mandell, J. E. Bennett, and R. Dolin. (New York: Churchill Livingstone), 2003-2009.

Sarkar-Tyson, M., Smither, S. J., Harding, S. V., Atkins, T. P., and Titball, R. W. (2009). Protective efficacy of heat-inactivated $B$. thailandensis, B. mallei or B. pseudomallei against experimental melioidosis and glanders. Vaccine 27, 4447-4451.

Schell, M. A., Ulrich, R. L., Ribot, W. J., Brueggemann, E. E., Hines, H. B., Chen, D., Lipscomb, L., Kim, H. S., Mrazek, J., Nierman, W. C., and Deshazer, D. (2007). Type VI secretion is a major virulence determinant in Burkholderia mallei. Mol. Microbiol. 64, 1466-1485.

Sharrer, G. T. (1995). The great glanders epizootic, 1861-1866: a civil war legacy. Agric. Hist. 69, 79-97.

Srinivasan, A., Kraus, C. N., Deshazer, D., Becker, P. M., Dick, J. D., Spacek, L., Bartlett, J. G., Byrne, W. R., and Thomas, D. L. (2001). Glanders in a military research microbiologist. $N$. Engl. J. Med. 345, 256-258.

Voisey, C. R., and Marincs, F. (1998). Elimination of internal restriction enzyme sites from a bacterial luminescence (luxCDABE) operon. Biotechniques 24, 56, 58.

White, N. J. (2003). Melioidosis. Lancet 361, 1715-1722.

Whitlock, G. C., Estes, D. M., and Torres, A. G. (2007). Glanders: off to the races with Burkholderia mallei. FEMS Microbiol. Lett. 277, 115-122.

Whitlock, G. C., Lukaszewski, R. A., Judy, B. M., Paessler, S., Torres, A. G., and Estes, D. M. (2008). Host immunity in the protective response to vaccination with heat-killed Burkholderia mallei. BMCImmunol. 9, 55. doi: 10.1186/1471-2172-9-55

Whitlock, G. C., Valbuena, G. A., Popov, V. L., Judy, B. M., Estes, D. M., and Torres, A. G. (2009). Burkholderia mallei cellular interactions in a respiratory cell model. J. Med. Microbiol. $58,554-562$.

Winson, M. K., Swift, S., Hill, P. J. Sims, C. M., Griesmayr, G., Bycroft, B. W., Williams, P., and Stewart, G. S. (1998). Engineering the luxCD$\mathrm{ABE}$ genes from Photorhabdus luminescens to provide a bioluminescent reporter for constitutive and promoter probe plasmids and mini-Tn5 constructs. FEMS Microbiol. Lett. 163, 193-202.

Conflict of Interest Statement: The authors declare that the research was conducted in the absence of any commercial or financial relationships that could be construed as a potential conflict of interest.

Received: 30 April 2011; accepted: 08 August 2011; published online: 26 August 2011.

Citation: Massey S, Johnston K, Mott TM, Judy BM, Kvitko BH, Schweizer HP, Estes DM and Torres AG (2011) In vivo bioluminescence imaging of Burkholderia mallei respiratory infection and treatment in the mouse model. Front. Microbio. 2:174. doi: 10.3389/fmicb.2011.00174

This article was submitted to Frontiers in Cellular and Infection Microbiology, a specialty of Frontiers in Microbiology. Copyright (c) 2011 Massey, Johnston, Mott, Judy, Kvitko, Schweizer, Estes and Torres. This is an open-access article subject to a non-exclusive license between the authors and Frontiers Media SA, which permits use, distribution and reproduction in other forums, provided the original authors and source are credited and other Frontiers conditions are complied with. 